\title{
IL PERSONALISMO DI JOSEPH RATZINGER/BENEDETTO XVI
}

\section{THE PERSONALISM OF JOSEPH RATZINGER/BENEDICT XVI}

\begin{abstract}
A b s t r a c t. Joseph Ratzinger, in his autobiography entitled My Life, writes about his encounter with personalism, later found explained by Martin Buber, as: "A spiritual experience that left an essential mark, even if I spontaneously associated such personalism with the thought of St. Augustin, who in his Confessions had struck me with the power of all his human passion and depth." From that moment on, all his theology emanates with personalism. He is convinced that "Christian faith does not relate to an idea but to a Person" (Intruduction to Christianity). The personalistic thinking of Joseph Ratzinger becomes evident when he describes the essence of the Sacrament of Baptism as well as the contents of the profession of faith in One and - at the same time - Three-personed God. "Being Christian is not the result of an ethical choice or a lofty idea, but the encounter with an event, a person" (Deus Caritas est, 1). Joseph Ratzinger-Benedict XVI underlines the significance of person in all his theology by focusing on the concept of person in Trinitology and Christology, in which he develops a personalism in the purest form.
\end{abstract}

Key words: personalism; person; God; faith; relationship.

Joseph Ratzinger nella sua autobiografia scrive: "L'incontro con il personalismo, che poi trovammo esplicito con grande forza persuasiva nel grande pensatore ebreo Martin Buber, fu un evento che segnò profondamente il mio cammino spirituale, anche se il personalismo, nel mio caso si legò quasi da sé con il pensiero di Agostino che, nelle Confessioni, mi venne incontro in tutta la sua passionalità e profondità umane"'.

Antonio Panaro, presbitero dell'Arcidiocesi di Varsavia, dottore in teologia dogmatica, formatore nel Seminario Arcidiocesano Missionario Redemptoris Mater e docente presso l'Accademia Cattolica di Varsavia; e-mail: apanaro78@gmail.com; ORCID: https://orcid.org/0000-0003-0048-9056.

1 Joseph Ratzinger/Benedetto XVI, La mia vita (Milano: San Paolo, 2013), 60-61. Vedi anche: Joseph RATZINGer, Aus meinem Leben. Erinnerungen (1927-1977) (Stuttgart: Deutsche Verlags-Anstalt,1998), 49. 
Prescindendo dal fatto che Joseph Ratzinger non venga menzionato fra i personalisti più conosciuti ${ }^{2}$, si può, tuttavia, tentare di sviluppare un'analisi da cui emergano i contenuti espressamente personalistici della sua teologia. Ratzinger non ha mai ha avuto l'intenzione di creare un sistema teologico, come ha più volte ribadito. È possibile, invece, trovare nella sua teologia chiare tracce di un personalismo propriamente ratzingeriano che, al tempo stesso, rispecchia in toto i canoni fissi di questa corrente di pensiero cristiana.

In che modo Ratzinger introduce il personalismo ${ }^{3}$ nella teologia ed in che consiste l'originalità della sua operazione? Sarà forse questa un'occasione per la teologia di presentare questioni antiche e ben conosciute con un nuovo linguaggio?

All'inizio, potremmo imbatterci in una serie di interrogativi o dubbi sulla scientificità di questo esperimento ratzingeriano, ossia riguardo l'introduzione, quasi a priori, del personalismo in teologia. Alcuni si chiedono, quanto possa essere radicato il pensiero teologico di Ratzinger nel personalismo, così da azzardare una tesi di questo tipo. In che consiste il concetto di persona elaborato da Joseph Ratzinger? Per l'ex-prefetto della Congregazione per la dottrina della fede, la questione riguardante la persona nella teologia ha un significato rilevante al fine di comprendere meglio ciò che costituisce l'essenza, il proprium dell'essere cristiani. Ratzinger ritiene che il concetto di persona, su cui esso si basa, sia uno dei contributi più validi, prodotti dal cristianesimo e dalla sua teologia, a favore del pensiero comune. All'origine del concetto di persona vi sono, infatti, due interrogativi fondamentali che costituiscono il centro della fede cristiana: chi è Dio (come ce Lo presenta la Bibbia)? e chi è Cristo? Per rispondere a questi due quesiti, il pensiero cristiano ha fatto uso del termine prosopon; termine totalmente sconosciuto al linguaggio filosofico fino a quel momento (II-III sec.). Lo stesso Ratzinger scrive del significato essenziale della persona nella teologia, in un intervento riportato poi nel suo libro Dogma und Verkündigung ${ }^{4}$.

Nell'Introduzione al cristianesimo inoltre aggiunge: "Ciò che è assolutamente unico, privo di relazioni e refrattario a qualsiasi rapporto, non potrebbe mai essere

2 Ecco alcuni tra i maggiori esponenti: Czesław Stanisław Bartnik, Mieczysław Gogacz, Stanisław W. Kowalczyk, Gabriel Marcel, Jacques Maritain, Emmanuel Mounier, Antoni Jozafat Nowak, Edith Stein, Tadeusz Styczeń, Pierre Teilhard de Chardin, Józef Tischner, Karol Wojtyła.

${ }^{3}$ Il personalismo è una corrente di pensiero prettamente di carattere filosofico.

${ }^{4}$ Joseph Ratzinger, Dogma und Verkündigung (Donauwörth: Erich Wewel Verlag, 2005), 201-219. Vedi anche Joseph RatzInger, Dogma e predicazione (Brescia: Editrice Queriniana, 2005), 173-189. Cf. Joseph Ratzinger, "Znaczenie osoby w teologii”, Personalizm nr 8(2005), 3548; cf. Bogumił GACKA, Znaczenie osoby w teologii Josepha Ratzingera-Benedykta XVI (Warszawa: UKSW, 2010). 
persona. Non esiste persona come entità singola e a sé stante, assolutamente isolata. [...] L'Assoluto è persona, non è affatto un'entità isolata; sicché il superamento del singolare è necessariamente incluso nel concetto di persona"s.

Per una corretta comprensione del pensiero espresso dal Papa teologo, va sottolineato che egli intende la persona in senso teologico e non filosofico. La Rivelazione mostra un Dio personale a livello esistenziale, e non sostanziale (essenziale). Nella filosofia greca la sostanza indicava l'essere come essere accanto ad un altro, sussistente e cioè indipendente nella propria esistenza. All'interno del Dio rivelato, le Persone sono relazioni sussistenti (relationes subsistens). Inoltre, il Dio della Bibbia si è fatto storia e si è rivelato all'interno della storia di salvezza. La filosofia antica non ha mai conosciuto la categoria dell'esistenza (subsistentia), ma solo quella della sostanza, essenza (essentia) ${ }^{6}$. "Le Persone in Dio sono Relazioni Sussistenti, Ipostasi, le quali non esistono a livello sostanziale $[\ldots]$, ma relazionale, di rapporto reciproco. La Santa Trinità costituisce una Comunione di Persone divine in un'unica e divina Natura, che di per Sé sono in relazione con Se Stesse"7.

La dottrina della Santissima Trinità è per il teologo bavarese un punto di partenza per sottolineare il carattere personale del Dio dei cristiani. Il Dio della fede differisce dal Dio dei filosofi, poiché è una Persona. Il Dio della Bibbia è un Dio personale e relazionale (cf. Gv 17,6.26).

Ecco il massimo paradosso della fede: il Dio dei padri, lontano e vicino al tempo stesso, distante ed accessibile. Il Dio della fede ha un nome, si presenta come numen personale (Dio di persone) e non come numen locale (divinità legata ad un luogo), "si rende nominabile". Il Logos - il Preesistente Pensiero Creatore di Dio si è fatto Persona, uomo e carne, conosce l'uomo ad intra nella sua umanità ed ama l'uomo, fino alla più alta donazione di Sé, morendo in croce e risuscitando?.

5 Joseph Ratzinger, Introduzione al cristianesimo (Brescia: Editrice Queriniana, 2013), 169-170.

${ }^{6}$ Cf. GaCKA, Znaczenie osoby, 16-17.

7 Ibidem, 17.

8 Cf. Ratzinger, Introduzione al cristianesimo, 113-117. Ne dà conferma la teologia veterotestamentaria riguardante il nome di Dio, nel Nuovo testamento invece il Vangelo di Giovanni. Il Dio della rivelazione "si rende nominabile, si abbandona agli uomini al punto che essi lo possano chiamare. E facendo questo, Egli entra in con-esistenza con gli uomini, lasciandosi da essi raggiungere". Giovanni ci presenta Cristo come "reale e vivo nome di Dio" (Ratzinger, Introduzione al cristianesimo, 113). "Ho manifestato il tuo nome agli uomini che mi hai dati, scelti di mezzo al mondo. [...] Ho fatto conoscere a loro il tuo nome e lo farò conoscere ancora, affinché l'amore col quale hai amato me, sia in essi ed io in loro (Gv 17,6.26)".

9 Ibidem, 126-127. Cf. Antonio Panaro, Wiara i Mysterium. Prymat Bożego daru w teologii Benedykta XVI (Lublin: Gaudium, 2014), 267-268. Cf. Gacka, Znaczenie osoby, 17. 
La cristologia di Joseph Ratzinger ha creato un personalismo dai connotati originali e, al tempo stesso, della miglior specie. Il personalismo di Ratzinger ha una dimensione preliminarmente cristologica: è legato alla Persona di Gesù Cristo ${ }^{10}$.

Dio in Cristo, facendosi uomo, pone Se Stesso in relazione "io-tu" con l'uomo. Egli è l'Emmanuele per eccellenza, il Dio con noi ed in noi. Poiché il Figlio procede dal Padre, ne consegue che Egli è un Essere in relazione "da - a" e "da - per" ", totalmente rivolto al di fuori di Se Stesso per amore all'uomo. Egli è disposto persino a compiere l'atto estremo di sacrificare la propria vita per l'uomo.

La struttura stessa del cristianesimo è profondamente personalistica: riguarda la mutua relazione delle Persone Divine con quelle umane. Il pensiero personalistico del Papa emerito emerge, quando analizza l'essenza del Sacramento del Battesimo come anche il Credo apostolico ${ }^{12}$. Attraverso il Battesimo i catecumeni entrano a far parte di una comunione sponsale con Dio talmente profonda, la cui espressione è il nome nuovo che ricevono. Rinuncia egli a essere un soggetto isolato ed un "io autonomo" per ritrovare se stesso nel nuovo "Io" di Cristo. Ratzinger afferma che il battesimo è vero sacramento di risurrezione per quanto prima lo sia di morte (Mt 10,39; Gv 12,24). San Paolo descrive la trasfigurazione esistenziale che avviene nel Battesimo, affermando: "Non sono più io che vivo, ma Cristo che vive in me (Gal 2,20)". Ratzinger a proposito osserva che il diventare "uno in Cristo Gesù (Gal 3,28)" indica proprio uno scambio ed una fusione dei soggetti nell'unica esistenza di Cristo. Non è il singolo che realizza il salvifico scambio, ma Cristo; il soggetto riceve passivamente "dal di fuori" e "da un Altro (Cristo)" il suo nuovo essere. La struttura sacramentale rivela l'esperienza mistica del primato del dono gratuito realizzato nell'evento ecclesiale del sacramento del Battesimo. La Chiesa, nella quale il neofita viene incorporato storicamente rende palese l'attiva comunione del "noi" dei credenti in Cristo nella quale si trasfigura l'io passivo del singolo, accolto da un "noi" che lo trascende ${ }^{13}$.

\footnotetext{
${ }^{10}$ Benedetto XVI, „Z wielką miłością do Chrystusa i Kościoła”, L’Osservatore Romano nr 26(2005), f. 7-8, 15.

${ }^{11}$ Cf. Ratzinger, Introduzione al cristianesimo, 176-178.

${ }^{12}$ Cf. Panaro, Wiara i Mysterium, 243-258, 270-294.

${ }^{13} \mathrm{Cf}$. ibidem, 251-253.
} 


\section{IL SIGNIFICATO DI PERSONA NELLA TEOLOGIA ${ }^{14}$}

"Il concetto di persona, e l'idea che gli sta dietro, è un prodotto della teologia cristiana $[\ldots]$, sviluppato soprattutto dalla discussione tra il pensiero umano e i dati della fede cristiana e su questa strada è entrato nella storia del pensiero"15. Come direbbe Etienne Gilson, il personalismo è uno dei "contributi" proposti e realizzati dalla fede cristiana a beneficio del pensiero umano comune. Questo tipo di contenuti per onestà e rigore scientifico (questo va ammesso) "non sorsero spontaneamente dal filosofare proprio dell'uomo", ma "dalla discussione tra la filosofia e le affermazioni della fede, e in particolare della Sacra Scrittura"16.

Poiché Il concetto di persona nasce fondamentalmente dai due interrogativi già esposti: chi è il Dio della Bibbia e chi è Gesù Cristo, il cristianesimo vi ha risposto (è questo il parere di Ratzinger) coniando il termine "persona" (dal greco prosopon) e forgiando concetti fino ad allora inesistenti ${ }^{17}$.

Ratzinger dice che il concetto di persona non può essere afferrato "se non viene di continuo ricongiunto" alla sua "origine" 18 : il credo dei cristiani ed in particolare la questione riguardante Dio e Cristo.

Infatti, nel corso dei secoli, "ci si è allontanati di molto" da questa origine, sviluppando una riflessione al di là della fede cristiana e del cristianesimo. Bisogna ammettere che il concetto di persona "trae vita" solo dalla fede - dice Ratzinger, anche se non ce n'accorgiamo ${ }^{19}$.

\section{IL CONCETTO DI PERSONA NELLA DOTTRINA DI DIO}

Per quanto concerne il concetto di persona e la sua evoluzione nel contesto della dottrina di Dio, Ratzinger li sintetizza elencando tre livelli che indicano un ulteriore approfondimento della riflessione in merito ${ }^{20}$ :

\footnotetext{
${ }^{14}$ Relazione ad un congresso sulla comprensione di persona nella pedagogia e nelle scienze ad essa vicine. Joseph Ratzinger, Dogma und Verkündigung (Donauwörth: Erich Wewel Verlag, 2005), 201-219; RAtZinger, Dogma e predicazione, 173-189 (trad. italiana). Cf. RATZINGer, „Znaczenie osoby w teologii”, 35-48 (tt. polskie).

${ }^{15}$ RatZinger, Dogma e predicazione, 173.

${ }^{16}$ Ibidem, 173.

${ }^{17}$ Ibidem, 173.

${ }^{18}$ Ibidem, 174.

${ }^{19}$ Ibidem, 174.

${ }^{20}$ L'autore ha scelto di chiamarli in questo modo per facilitare l'esposizione. Ratzinger invece non dà nessun titolo.
} 
(1) Stadio esegetico-lessicale: Tertulliano (155 ca.-233 ca.) conia il termine latino persona. Nasce la famosa formula: Dio è "una substantia - tres personae"21.

(2) Stadio teologico-trinitario: consiste principalmente nell'affermare che l'essere in relazione è un elemento fondante e costituente della persona stessa. Essa va intesa di per sé come "relazione" (puro esistere in riferimento a qualcun altro) e si sottolinea che questo non è un fattore aggiuntivo.

(3) Stadio antropologico: il concetto di persona come relazione va esteso (quasi per riflesso) a tutto lo spirito umano. La sua origine trinitaria è "portante"22, ma non lo preclude unicamente alla realtà della Santa Trinità. Esso si apre alla realtà di ogni uomo (ciò ha radicali conseguenze di tipo antropologico).

(4) Stadio esegetico-lessicale. Anzitutto non si può nascondere la meraviglia e lo stupore per la genialità di Tertulliano, nell'aver dato una definizione precisa ed aver coniato il termine persona, a cui ha fatto seguito tutta una terminologia fino ad oggi valida. Ratzinger commenta ciò dicendo che questa storia ha un alcunché di "irreale"23. Uno studio di Carl Andersen di Gottinga (1961), esperto in storia dei dogmi, risponde comunque alla domanda su come il cristianesimo sia arrivato ad una tale risposta. Andersen afferma che gli scrittori cristiani abbiano fatto uso nella loro lettura esegetica della Sacra Scrittura, di un artifizio letterario, già conosciuto nella letteratura antica, chiamato esegesi prosopografica. Essa è una sorta di interpretazione del testo, che permette di riconoscere le varie persone che l'autore faceva entrare in scena nel racconto e che dialogavano tra di loro. Il fine dell'utilizzo di questa figura letteraria era quello di ravvivare drammaticamente gli eventi descritti ${ }^{24}$. Gli autori cristiani, a differenza dei poeti pagani, utilizzano l'esegesi prosopografica attribuendo sin dal II sec. al termine prosopon il significato di persona e non di ruolo; per cui i ruoli di cui parla l'autore sacro sono realtà ed entità in dialogo. Vediamo come è evoluta l'idea di persona nel contesto dell'esegesi cristiana ${ }^{25}$.

(5) Stadio teologico-trinitario è lo stadio successivo, che presenta come sia maturato il concetto di persona all'interno della teologia, a distanza di circa duecento anni più tardi (alla fine del IV e agli inizi del V sec.). Ora viene affermato con certezza dogmatica che la persona deve essere intesa come relazione: "Le tre persone che esistono in Dio sono, per loro natura - come dicono Agostino e la teologia del-

\footnotetext{
${ }^{21}$ Ratzinger ricorda che la formula definitiva dell'occidente è "una essentia tres personae" (Agostino). Cf. Ratzinger, Dogma e predicazione, 174.

${ }^{22}$ Ibidem, 181.

${ }^{23}$ Ibidem, 175.

${ }^{24}$ Prosopon dal greco significa anzitutto "ruolo, maschera dell'attore"; solo in seguito esso ha acquisito il significato esplicito di persona (Ibidem, 175).

${ }^{25}$ Ibidem, 177.
} 
la tarda patristica - relazioni, rapporti. [...] La relazione, l'essere riferimento, non è qualcosa di aggiuntivo alla persona, ma è la persona stessa" ${ }^{26}$. Ratzinger afferma che la Sacra Scrittura e precisamente la teologia giovannea evidenziano "questo fenomeno della pura relatività". "Il Figlio da Sé non può far nulla (cf. Gv 5,19)", poiché con il Padre sono uno (cf. Gv 10, 30), il che equivale a dire, che il Figlio non si pone accanto al Padre, come una sostanza ben definita, ma esiste in totale relazione con il Padre, non riservando a se stesso alcuna "proprietà personale". Lo stesso principio vale per la relazione tra i discepoli e Cristo, tra l'uomo e Dio: "Senza di me non potete far nulla (Gv 15,5) e prega perché siano "una cosa sola", come Lui ed il Padre (Gv 17,1) ${ }^{27}$. La relazione con Cristo diventa "la parte essenziale dell'esistenza dei discepoli" ${ }^{28}$.

(6) Stadio antropologico: l'uomo ad immagine della Santissima Trinità è essere relazionale e lo si può comprendere partendo da questo concetto di Dio. A differenza di Agostino, Ratzinger non termina l'analisi al rapporto tra la Santa Trinità e l'uomo, ma parla di un concetto di uomo aperto verso l'altro uomo. Che parte da un input iniziale trinitario ed è diretto verso l'altro uomo e il totalmente Altro, che è Dio Stesso. L'uomo cioè tende in Cristo verso il completamento definitivo di sé in Dio. Ecco un nuovo tema da introdurre: la persona nella cristologia.

\section{IL CONCETTO DI PERSONA NELLA CRISTOLOGIA}

Proviamo a rispondere all'enigma: chi e che cosa è Cristo? "La teologia ha risposto con la formula: Egli ha due nature ed una persona, natura divina ed umana, ma soltanto una persona divina" ${ }^{29}$.

Ratzinger, affrontando la suddetta questione, dapprima evidenzia e risolve due malintesi e facili fraintendimenti del dogma:

(1) dire che "Cristo ha soltanto una persona" non significa declassare, sminuire, non è una sottrazione della completa umanità di Gesù ${ }^{30}$. La storia dei dogmi è una grande testimonianza di lotta "palmo a palmo" a difesa di questa realtà ${ }^{31}$. Invece,

${ }^{26}$ Ibidem, 178.

${ }^{27}$ Ibidem, 180.

${ }^{28}$ Ibidem, 180.

${ }^{29}$ Ibidem, 182.

${ }^{30}$ Ibidem, 182

${ }^{31}$ Ibidem, 182. Le seguenti eresie declassavano in qualche aspetto Cristo come persona: l'arianesimo, l'apollinarismo ritenevano che Cristo non avesse avuto un'anima; il monofisismo Gli negava la natura umana; il monoteletismo Lo vedeva privo della volontà umana; il monenergismo ammetteva la sua volontà umana che nell'attualizzazione però veniva sostituita da quella divina. 
proprio questo concetto cristologico di persona va riferito ad ogni singolo uomo; è questo "il contributo della fede cristiana alla totalità del pensiero umano"32;

(2) è errata la tesi, secondo la quale, Cristo sia un'eccezione ontologica del tutto particolare a differenza del resto degli uomini. Di conseguenza, Egli viene escluso così dalle regole dell'umano e del naturale ${ }^{33}$. Cristo invece è secondo Ratzinger: "lo specifico compimento dell'idea di uomo"; è "la rivelazione di tutta l'essenza dell'uomo" e della massima realizzazione ${ }^{34}$.

\section{RISPOSTA POSITIVA DI RATZINGER AL DOGMA CRISTOLOGICO}

Eliminati i due malintesi bisogna - a dire di Ratzinger - stabilire, cosa significa positivamente la formula: "Cristo ha due nature in un'unica persona" e dopo aver ammesso che in questo caso la risposta teologica non è ancora completamente matura, afferma di poter tentare solo di abbozzare una risposta ${ }^{35}$.

Ratzinger fondamentalmente presenta tre questioni:

1. "l'essenza dello spirito in generale è l'essere-in-relazione";

2. la formula cristologica ${ }^{36}$ va intesa come un indicazione direzionale;

3. l'idea del "noi" ha prima di tutto una valenza ontologica trinitaria anziché solo antropologica.

\section{L'essenza dello spirito in generale è l'essere in relazione}

Ratzinger spiega tale affermazione in questo modo: la persona non soltanto esiste, ma è per natura chiamata a superare se stessa guardando all'altro e nel rapporto con l'altro, in questo senso ritorna su se stessa, possiede se stessa. "L'esserepresso-l'altro è il suo modo d'essere presso se stesso"37. Il Vangelo ne dà conferma quando dice: "Chi avrà perduto la propria vita per causa mia, la troverà (Mt 10,39). La natura redenta da Cristo in Lui ritrova il suo modo di essere vero, quello di essere presso l'altro perdendo apparentemente se stessa, ma ritrovandosi in se stessa proprio nell'atto del relazionarsi.

${ }^{32}$ Ibidem, 182.

${ }^{33}$ Ibidem, 184.

${ }^{34}$ Ibidem, 184-185.

35 "Posso tentare soltanto di fornire qualcosa come un abbozzo", quasi a voler "indicare la direzione", verso la quale si deve procedere per effettuare un'analisi credibile in merito (Ibidem, 185).

${ }^{36}$ Si tratta della formula: "una persona duae naturae".

${ }^{37}$ RatZinger, Dogma e predicazione, 186. 


\section{La formula cristologica "una persona duae naturae" va intesa come un vettore}

Indicazione, "una freccia direzionale" 38 per l'uomo in generale che lo conduce verso il compimento pieno della sua umanità. Cristo, nuovo Adamo ha iscritto nella natura umana la forma più radicale e dinamica dell'esistenza, che consiste nell'essere presso l'altro. "In Cristo, l'uomo che è pienamente accanto a Dio, non viene annullato" nel suo essere uomo, "ma giunge invece alla sua più alta possibilità, che consiste nell'andare oltre se stesso, verso l'Assoluto e nel raggiungere" [...], il totalmente altro, cioè l'assolutezza dell'amore divino"39.

\section{Il "Noi" è anzitutto intra-trinitario, prima che extra-trinitario}

Il cristianesimo ha stravolto il pensiero comune antico che definiva Dio come "pura monarchia dell'unicità e singolarità. [...] Il concetto cristiano di Dio ha dato, per principio, identica dignità sia alla molteplicità che all'unicità. Mentre per gli antichi la molteplicità appare soltanto come la dissoluzione dell'unità, per la fede cristiana, che ragiona in termini trinitari, la molteplicità possiede la stessa dignità dell'unicità"40. L'io divino e quello umano sono nascosti per entrambe le parti in un "Noi" più grande sia trinitario che umano ${ }^{41}$. Ratzinger osserva che non prendere in considerazione questo noi ha influenzato radicalmente il concetto di Chiesa ridotta ad una dimensione meramente individualista e non comunitaria ${ }^{42}$.

Concludendo le nostre riflessioni sul personalismo di Joseph Ratzinger, vediamo come grazie all'interpretazione personalistica della Santissima Trinità, affiora una nuova comprensione della realtà dell'uomo e di Dio. Paradossalmente dobbiamo convenire con Ratzinger ed affermare che "parlando di Dio si intravvede chi sia 1'uomo", ciò che sembra paradossale "è più chiaro e più di aiuto"43.

\section{BIBLIOGRAFIA}

Agostino. Commento al vangelo e alla prima epistola di san Giovanni. In Opere di Sant'Agostino XXIV/1 (Roma: Città Nuova, 1968).

BenEDYKT XVI. “Z wielką miłością do Chrystusa i Kościoła”. L'Osservatore Romano nr 26(2005), f. 7-8, 15.

\footnotetext{
${ }^{38}$ Ibidem, 187.

${ }^{39}$ Ibidem, 187.

${ }^{40}$ Ibidem, 188.

${ }^{41}$ Ibidem, 188.

42 Ibidem, 188.

${ }^{43}$ Ibidem, 180.
} 
RatZinger, Joseph/Benedetto XVI. La mia vita. Milano: San Paolo, 2013.

RatZINGER, Joseph. Aus meinem Leben. Erinnerungen (1927-1977). Stuttgart: Deutsche Verlags-Anstalt, 1998.

RATZINGER, Joseph. Introduzione al cristianesimo. Brescia: Editrice Queriniana, 2013.

Ratzinger, Joseph. Dogma und Verkündigung. Donauwörth: Erich Wewel Verlag, 2005.

RatZinger, Joseph. Dogma e predicazione. Brescia: Editrice Queriniana, 2005.

RatZinger, Joseph. „Znaczenie osoby w teologii”. Personalizm nr 8(2005), 35-48.

Panaro, Antonio. Wiara i Mysterium. Prymat Bożego daru w teologii Benedykta XVI. Lublin: Gaudium, 2014.

GACKA, Bogumił. Znaczenie osoby w teologii Josepha Ratzingera-Benedykta XVI. Warszawa: UKSW, 2010.

\section{PERSONALIZM JOSEPHA RATZINGERA/BENEDYKTA XVI}

S TRE S Z C Z E NIE

Joseph Ratzinger w autobiografii zatytułowanej Moje życie pisze o swoim spotkaniu z personalizmem, który potem odnalazł jako jasno wyeksponowany u Marcina Bubera: „Stało się [spotkanie z personalizmem] dla mnie istotnym duchowym przeżyciem. Personalizm ten kojarzył mi się mimowolnie z myślą Augustyna, który w Wyznaniach wyszedł mi niejako naprzeciw z całą swoją ludzką namiętnością i głębią". Od tamtego momentu cała teologia Ratzingera zaczęła emanować personalizmem. Jest on przekonany, że „Wiara chrześcijańska nie przedstawia jakiejś idei, lecz Osobę” (Wprowadzenie w chrześcijaństwo). Personalistyczne myślenie Josepha Ratzingera uwypukla się bardziej, gdy Ratzinger analizuje sakrament chrztu oraz treść wyznania wiary w Jednego a zarazem trójosobowego Boga. „U początku bycia chrześcijaninem nie ma decyzji etycznej czy jakiejś idei, ale natomiast spotkanie z wydarzeniem, z Osobą" (Deus Caritas est 1). Joseph Ratzinger/ Benedykt XVI podkreśla znaczenie osoby w całej swojej teologii. Przedstawia on jednak personalizm w najczystszej postaci, szczególnie kiedy bada koncepcję osoby pod kątem trynitologicznym i chrystologicznym.

Słowa kluczowe: personalizm; osoba; Bóg; wiara; relacyjność. 